\title{
Causal Reasoning in Physics
}

\author{
Mathias Frisch \\ University of Maryland, College Park \\ mfrisch@umd.edu
}

\section{Introduction ${ }^{1}$}

It appears to be both natural and intuitive to think of the world as causally evolving. We conceive of events in the present as being caused by events in the past and, in turn, as acting as causes for what happens in the future. But it is also a widespread view - at least among philosophers of physics - that this conception is not part of how mature physics represents the world. According to this view, the notion of cause survives - if at all — as part of a 'folk' scientific conception of the world but has no place in our mature theories of physics. In this paper I want to examine critically a cluster of arguments in favor of this causal skepticism, focusing in particular on the asymmetry of the causal relation, and I will try to defend the view that causal notions play an important role at least in some mature physical theories. Rich causal notions, I want to maintain, are an integral part of how physicists represent the world within the context of some mature theories and causal reasoning plays an important role even in contemporary physics. A large part of this paper will be devoted to fending of arguments advanced by causal skeptics, but I will also discuss a case study that provides positive evidence in support of my claim.

In the next section I will distinguish several dimensions of our intuitive, pre-philosophical notion of cause that are invoked in the context of scientific theorizing. One core aspect of the notion of cause is the asymmetry of the causal relation and this aspect will be the main focus of this paper. In section three I will critically examine a range of arguments aimed at showing that there is no room for an asymmetric causal relation in mature physics. Many of these arguments are descendents of Bertrand Russell's famous attack on the notion of cause (Russell 1918), but they also have found more recent defenders (see, for example, van Fraassen 1993, Norton 2007, forthcoming; Hitchcock 2007). ${ }^{2}$ In section four I will discuss an example of the use of causal notions in physical theorizingthe appeal to an asymmetric causal constraint in the derivation of both classical and quantummechanical dispersion relations. I will end with a brief conclusion.

It may be helpful at the outset to contrast the view in which I am interested with the view that John Norton criticizes in (Norton 2007) and (Norton forthcoming). While some of Norton's

\footnotetext{
${ }^{1}$ Ancestors and earlier versions of this paper have been presented at the Boston Colloquium in 2006, a workshop at the University of North Carolina, Chapel Hill in 2006, the conference "Causation and Mechanism" at the University of Maryland in 2007, and the $1^{\text {st }}$ conference of the European Philosophy of Science Association in Madrid in 2007. I want to thank the audiences there for extremely useful comments and criticisms.

2 As I learned only recently, (Steiner 1986) argues for a conclusion quite similar to mine, also using Russell's discussion as his main target. But Steiner's argument centrally relies on certain interpretive assumptions about quantum mechanics, while my defense of causal notions makes no such assumptions.
} 
arguments are meant to show that the notion of cause is merely part of 'folk science' and hence are directed at the thesis I will be defending, his criticisms are also aimed at a stronger claim: the view he calls "causal fundamentalism" and which maintains that "nature is governed by cause and effect and the burden of individual sciences is to find the particular expression of the general notion in the realm of their specialized subject matter" (Norton 2007, 15). My thesis is not committed to either of the two conjuncts of causal fundamentalism, as defined by Norton. It is not committed to the first conjunct, since it allows for the possibility that on the most fundamental level—whatever that may be — nature is not governed by cause and effect, even though there are certain mature sciences which involve causal representations of nature. And it is not committed to the second conjunct, because the thesis allows that even if nature were fundamentally governed by cause and effect, it need not be the job of each and every individual science to find expressions of this notion. There may be many fruitful ways of representing various parts of nature that do not involve causal notions. Thus, I will not here argue against Norton's weaker thesis - the claim that causal fundamentalism is false-but only against the stronger claim that the notion of cause is at best part of a folk science and plays no role in physics.

Norton argues that to the extent that causal notions are present in physics they play only one of two roles. First, causal notions may serve as labels used to classify different classes of solutions to a theory without however restricting the theory's factual content (see, e.g., Norton forthoming, 224). Second, causal notions may play a more substantive role in certain domain-restricted nonfundamental theories, but in all such cases, Norton maintains, the putatively causal relations can always be understood as being generated from more fundamental non-causal laws through reduction relations, similar to the case of the notion of 'caloric' (see, e.g., Norton 2007, 42). For the purposes of this paper I want to remain agnostic on the question whether all uses of causal notions in scientific theorizing are ultimately reducible to more fundamental, non-causal notions. I do not know of any cogent argument that can show that Norton is correct and that all occurrences of substantive causal notions are reducible, but I also have no argument that this is in principle impossible. I do, however, want to defend the thesis that there are instances where such notions play a more substantial role than that of an empty honorific.

\section{Causal Dimensions}

Russell famously claimed that the word 'cause' is not used in the advanced sciences. Yet, as has been pointed out repeatedly — for example by Patrick Suppes (Suppes 1970) and more recently by Chris Hitchcock (Hitchcock 2007) — this claim can easily be shown to be false. Contrary to what Russell maintained, the words 'cause', 'causal', and related words are still widely used in contemporary physics. But what do physicists mean when they use causal language and what are their reasons for invoking causal notions? Unfortunately these questions do not permit of a simple answer, since the 
use of causal notions is not much more well-regimented in science than it is in everyday $\operatorname{life}^{3}$ and causal terms are used to express a variety of different claims in a variety of different contexts. There is a growing literature arguing that our intuitive notion of cause is a cluster concept and that several distinct notions are lumped together in our common sense notion of cause. Independently of whether this claim is correct or whether there is a unique relation that is picked out by paradigmatic uses of causal language, clearly there are a variety of different aspects or dimensions to our employment of causal language, and causal talk in physics reflects these different aspects. Three dimensions, appear to play, or have played, a particularly important role in physical theorizing.

The first dimension is that effects covary with their causes or that effects are determined by their causes. It used to be common to think of causes as sufficient conditions for their effects or at least as parts of sufficient conditions and the notion of cause was seen to be closely connected to a condition of determinism - so closely, in fact, that physicists, such as Hermann Weyl, have referred to a principle of determinism as "the law of causality" (Weyl 1989, 40). Weyl distinguished this notion of cause from a second notion that he referred to as "the metaphysical notion of 'the reason for something”" (Weyl 1989, 31). Similar to Weyl's first notion, Niels Bohr said that "in physics, causal description [...] rests on the assumption that the knowledge of the state of a material subsystem at a given time permits the prediction of its state at any subsequent time" (Bohr 1948, 312); and Erwin Schrödinger called the "principle of causality" the requirement that "the exact situation at any point $P$ at a given moment is unambiguously determined by the exact physical situation within a certain surrounding of $P$ at any previous time, say $t-\tau$." (Schrödinger 1951,28) Schrödinger's principle of causality, in fact, combines two dimension of the notion of cause - a condition of determinism with a locality condition - but for him, as for Weyl and for Boh,r the fact that a theory is causal (in one sense) implies that it is deterministic. Thus, quantum mechanics was taken to pose a threat to causality simply because it is an indeterministic theory.

Today, however, it seems that the connection between causation and determinism is generally seen to be less tight. We have apparently learned to live with genuine indeterminism and, correspondingly, our notion of cause has broadened to allow for the possibility of probabilistic causation. Thus, it seems to be less common among physicists today than it was perhaps in the first half of the twentieth century to refer to a condition of determinism as 'principle' or 'law of causality' and causal notions are more commonly employed in other contexts.

A second common and more contemporary usage of causal language in physics is to express various locality conditions. One cluster of such conditions concerns the demand, implicit in Schrödinger's principle of causality, that there be no gaps between causally related events- where the prohibition may be against spatial, temporal, or spatiotemporal gaps. ${ }^{4}$ Newtonian gravitational theory

\footnotetext{
3 One rare exception is Fritz Rohrlich's discussion of different causal notions in (Rohrlich 1990).

${ }^{4}$ Prohibitions against 'gappy' causation are discussed in (Lange 2002) and in (Frisch 2005).
} 
is acausal, according to such a criterion, since there is no medium transmitting gravitational influences, while classical electromagnetism, due to the presence of electromagnetic fields, is causal. Another, conceptually distinct type of constraint concerns the spatiotemporal distances between causally related events and the speed at which causal influences propagate. The main examples of this kind of constraint in contemporary physics are, of course, the constraints imposed on relativistic theories: first, the condition that there is a finite, invariant velocity - the velocity of light - a condition often expressed as demanding that spacetime has a lightcone structure; and, second, the condition that there is no propagation in matter faster than the speed of light. These two constraints and the spacetime structures satisfying them are often characterized in causal terms. For example, two points in spacetime that can be connected by a signal traveling at most at the speed of light - that is, points that are either timelike or lightlike related to each other-are called "causally connectable." And curves in spacetime representing points moving at less than or equal to the speed of light are called "causal curves." In general relativity models of Einstein's field equations are said to satisfy a "causality condition" if they do not contain closed causal curves. In quantum field theories relativistic constraints are implemented in the form of a condition called "micro-causality," which demands that the commutator between fields at spacelike separated spacetime points vanishes. Micro-causality is meant to capture the intuitive condition that the value of the field at one spacetime point can make no difference to the value of the field at another point, if the spacetime points are spacelike separated - that is, the two spacetime points could not be connected by a light signal or by any object moving slower than the speed of light.

One important usage of causal language, then, in the context of relativistic theories is to express time-symmetric locality constraints, such as that spacetime has a lightcone structure. But there is a second aspect to causal talk in relativity: causal notions are also used to mark a timeasymmetric distinction between the future lightcone, which is called "the causal future" of an event, and the past lightcone, which is the "causal past" of an event. This latter usage reflects the fact that the causal relation is asymmetric: if $c$ is a cause of $e$, then it is not the case that $e$ is a cause of $c$. This third aspect of the notion of cause - the causal asymmetry - is arguably the most central of the three dimensions and will be the main focus of this paper. The asymmetry is clearly an integral part of our intuitive idea that causes 'bring about' or 'produce' their effects, and also of Weyl's notion of the reason for something, but it is also an integral part of less metaphysically 'weighty' notions of cause. As the identification of the causal future with the future lightcone in relativity theory attests, the causal asymmetry is intimately related to a temporal asymmetry, even though what precisely the relation is, is somewhat of a delicate issue. On some accounts of causation, such as Humean regularity accounts, it is a conceptual truth that effects do not precede their causes, but even those accounts that allow for the conceptual possibility of backward causation would presumably maintain that causation in our world (or at least in the spatiotemporal region of the universe accessible to us) is forward directed, and hence, causal constraints are often taken to imply time-asymmetric constraints. 
In the next section I want to assess a number of arguments that aim to show that there is no room for a time-asymmetric notion of cause in physics. Many of the arguments appeal to the fact that the asymmetry is not reflected in the theories' fundamental equations. The only legitimate role an asymmetric notion of cause could play, if these arguments are successful, is the one Norton allows for causal notions in relativistic theories: as devices for labeling or cataloging different parts of a theory's models. On Norton's view, the fact that the future lightcone of an event is called its "causal future", has no implications of any physical significance. I shall argue, however, that the anti-causal arguments do not succeed and will show by means of a specific example that time-asymmetric causal principles can play a substantive role in physical theorizing.

\section{No place for asymmetric causes?}

Again I want to begin with Russell's famous paper. Russell argues there that imprecise common sense causal regularities are replaced in physics by precise laws that have the form of functional dependencies. Russell's argument appears to be roughly this. Putatively causal claims need to be underwritten by universal causal regularities of the form "All events of type $A$ are followed by events of type $B . "$ But in trying to find such regularities, we are faced with the following dilemma. Either the events in question are specified only vaguely and imprecisely. The resulting regularities might be multiply instantiated, but they are formulated too imprecisely to be properly scientific. Or the events in question are specified precisely, but then the resulting regularities are instantiated at most once. Physics avoids this dilemma by providing us with precise functional dependencies. That is, instead of vague regularities of the form "When an object at rest experiences a sufficiently strong force it tends to begin to move" or a precise but perhaps only singly instantiated regularity of the form "Whenever a ball of mass $m_{0}$ in circumstances $c_{0}$ is struck with a force $f_{0}$ it accelerates at rate $a_{0}$ " physics presents us with logically much stronger functional dependencies, such as that all massive bodies obey Newton's law $\boldsymbol{F}=m \boldsymbol{a}$. Russell, thus, claims that such functional dependencies have replaced putatively causal regularities in physics, but of course it does not follow from the fact that physical theories present us with functional dependencies that these dependencies themselves cannot be understood causally. How, then, might we try to establish the claim that there is no legitimate place for an asymmetric notion of cause in fundamental physics? I want to examine several arguments for this claim, some of which are meant to spell out some of Russell's own suggestions while others are suggested by the more recent literature.

I will begin with what may be the most ambitious argument — an argument that does not explicitly invoke the asymmetry of the notion of cause and is aimed at showing that any notion of cause that does not take causal relations to be reducible to non-causal facts is meaningless or incoherent. This argument is suggested, for example, by some of van Fraassen's discussion of causation and begins by asking us to contrast a putatively causal world with a 'Hume world' replica of that world - that is, a world, that is identical to the first world as far as its Humean matters of fact 
are concerned but that does not include any causal relations between events (see van Fraassen 1993). If we further assume that the Hume world is empirically indistinguishable from its putatively causal twin, and also assume a weak verification principle according to which for a concept to be meaningful, there must be some empirical differences between the situations when the concept applies and those when it does not, then it follows that the notion of causation is meaningless. Thus, the argument is:

1. If there are asymmetric causal relations between events in a world (a c-world), then there is a qualitatively distinct possible world that is a replica of the c-world except that there are no causal relations between any events in that world (a Hume-world replica).

2. A c-world and its Hume-world replica are qualitatively distinct, only if the notion of cause is meaningful.

3. For the notion of cause to be meaningful, a c-world and its Hume-world replica must be empirically distinguishable, at least in principle. (Verification principle)

4. The c-world and its Hume-world replica are empirically indistinguishable.

5. Therefore, the c-world and its Hume-world replica are not qualitatively distinct.

6. Therefore, the c-world contains no asymmetric causal relations. $(1,5)$

Note that this argument does not, of course, undermine any account of causation that takes causal relations to be reducible to Humean facts, since such accounts would deny (1). Any world containing the same Humean matters of fact would contain the same causal facts, if causal facts were reducible to non-causal facts. Yet advocates of a Humean account of causation are not the only ones who would reject (1). A defender of a notion of cause richer than that allowed by a Humean can question whether the notion of a Hume world replica is coherent. Thus, Nancy Cartwright has argued that many events, such as shovings or milk lappings, are intrinsically causal (Cartwright, 1993, 427). If this is correct, then a Hume-world replica of a causal world, in which events have all their noncausal properties but none of their causal properties, does not constitute a coherent possibility. Imagining a world that is like ours except for its causal content - that is, among other things, a world in which the cat's tongue moves as it does in the actual world and the milk disappears in its mouthbut in which there are no intrinsically causal lappings, Cartwright contends, "is ridiculous." (427) Thus, a defender of causation could, with Cartwright, reject (1).

It is not easy to assess the relative merits of these two competing positions here in ways that do not simply beg the question against one of the two views. Both the defender of causation and the causal critic want to conclude that a c-world is not qualitatively distinct from its Hume-world replica. According to the argument of the causal critic, a putatively causal world already is a Hume world, while the defender of causation insists that any replica of a causal world will still contain causal relations, if that world is to present a coherent possibility at all. Is it not possible to coherently 
imagine qualitatively distinct pairs of c-worlds and Hume worlds, because the notion of cause itself is incoherent or because causal relations are woven into the very fabric of causal worlds in ways that makes it impossible coherently to imagine their absence? I worry that at this point we have simply reached an impasse of clashing intuitions.

Our first argument does not yet involve any appeal to physical theorizing. Perhaps we can sidestep the issue concerning the coherence of a Hume world and do so in a way that also explicitly includes an appeal to mature theories of physics. For a concept to be meaningful we might assume, there have to be conditions of its employment that are ultimately underwritten by the results of science — science, that is, is the ultimate arbiter of meaningfulness. But, the argument contends, causal notions are not part of mature physics:

7. For the notion of cause to be meaningful, it has be part of the mature theories of physics. (Verification principle*)

8. The notion of cause is not part of any mature physical theory.

9. Therefore, the notion of cause is not meaningful. $(7,8)$

A serious problem with this argument is that-like the previous argument-it relies on a verification principle of meaning and the history of twentieth century philosophy has taught us how dubious such principles are. Moreover, (7) is even more questionable than its cousin (3), since there obviously are many meaningful concepts that are not part of mature physics. Also, the argument's second premise, premise (8), is itself in need of an argument. Thus, it seems more promising to grant the meaningfulness of an asymmetric notion of cause and argue against the claim that such a notion has a legitimate role to play in physical theorizing. In what follows, then, I want to example possible arguments for (8), restricting myself to arguments that do not rely on the claim that causal notions are meaningless.

Russell suggests one argument for (8) during his discussion of Newton's law of gravity. He says that "in the motion of mutually gravitating bodies, there is nothing that can be called a cause and nothing that can be called an effect; there is merely a formula." (141, my emphasis) This remark is echoed by Bas van Fraassen, who answers Cartwright's question "why not allow causings in the models?" as follows:

To me the question is moot. The reason is that, as far as I can seen, the models which scientists offer us contain no structure which we can describe as putatively representing causing, or as distinguishing causings and similar events which are not causings. [...] Some models of group theory contain parts representing shovings of kid brothers by big sisters, but group theory does not provide the wherewithall to distinguish those from shovings of bog sisters by kid brothers. The distinction is made outside the theory. (van Fraassen, 1993, 437-8)

While Russell's remark suggests that a theory ought to be strictly identified with a set of formulas, van Fraassen argues that a theory consists of a set of state-space models. But even though the two 
disagree on whether theories ought to be understood syntactically or semantically, they agree that there is no place for causal notions in physical theorizing. I think that we can reconstruct their remarks in terms of the following argument:

10. The content of a physical theory is exhausted by a set of state-space models or a set of formulas.

11. Causal relations are not part of the formulas or models of a theory.

12. Therefore, causal relations are not part of the content of physical theories.

As it stands, however, (10) is false. Mathematical physics provides us with mathematical models or representations of the world, yet on their own mathematical models do not represent anything. How a given model or class of models represents the world depends on how the model is interpreted. Thus, no theory of physics can be strictly identified with a set of formulas or state-space models, since, minimally, a theory has to contain an interpretation which tells us which bits of the formalism are hooked up with which bits of the world. Minimally, a theory's interpretation has to specify the theory's ontology. ${ }^{5}$ But once we see that the austere view of theories as consisting solely in a mathematical formalism or set of models is untenable and that an interpretive framework needs to be part of a theory, it is no longer obvious that this framework cannot be rich enough to include causal assumptions as well. That is, we cannot conclude from the mere fact that an uninterpreted formula does not on its own mark, say, F's as causes and a's as effect, that one cannot interpret the formalism causally.

One common suggestion is that the specific form of the fundamental equations of physics entails that these equations cannot be interpreted causally. These equations, it is argued, are timesymmetric and, hence, do not permit of an asymmetric causal interpretation. Again, this view can be traced to a remark by Russell, who said that "the laws make no difference between past and future: the future 'determines' the past in exactly the same sense in which the past 'determines' the future." One way to interpret Russell here is as claiming that the purported fact that physics provides us with time-symmetric functional dependencies undermines the claim that asymmetric causal relations can play a role in physics. On another reading, the premise of the argument is that the fundamental equations are both future- and past-deterministic - that is, define both a well-posed initial and a wellposed final value problem - irrespective of whether the laws are time-symmetric or not. These two readings result in two distinct arguments. First, the argument from determinism:

13. The fundamental equations of classical physics are both past- and futuredeterministic.

\footnotetext{
${ }^{5}$ One might think that on van Fraassen's view (or at least according to a view van Fraassen once held) a theory does not require an interpretational framework. On that view a theory is true, if it has models that are isomorphic to the phenomena. The problem with that view, however, is that there may be too many isomorphisms and hence that almost all theories come out as (almost) trivially true.
} 
14. There is no place for an asymmetric notion of cause in the context of a theory with fundamental equations that are both past- and future-deterministic.

15. Therefore, there is no place for an asymmetric notion of cause in mature physical theories.

Of course, we no longer believe that the fundamental laws of nature are deterministic, but there are independent reasons for rejecting the conclusion. The argument relies on the assumption that in situations where causes determine their effects, the set of effects of an event cannot in turn determine its causes, and this premise does not appear to be defensible. Mackie's INUS condition account, for example, has the consequence that under some very weak additional assumptions, effects are also INUS conditions of their causes. But it does not follow from this fact, that it is impossible to supplement Mackie's account with some condition that allows us asymmetrically do distinguish causes from effects. More generally, it is hard to see why the notion of an event asymmetrically causing certain effects should be incompatible with the effects determining the occurrence of their causes. The claim cause that causes in some sense bring about their effects does not seem to preclude the possibility that the occurrence of certain evens can be used to infer the occurrences of their causes.

Once we move to the context of genuinely probabilistic theories, the case of the defender of causal relations may be even stronger, since, as (Callender 2000) shows, any non-trivial theory that specifies transition probabilities possesses a time-asymmetry. Any theory that specifies both nontrivial forward and on-trivial backward transition probabilities for a system has the consequence that the expected state of the system cannot change with time. Hence, any interesting physical theory could specify forward transition probabilities or backward transition probabilities, but not both. In particular, this argument shows that a quantum theory with transition probabilities cannot be timereversal invariant. A proponent of a causal interpretation of a probabilistic theory might appeal to this asymmetry and argue that the direction of causation ought to be identified with the direction of the theory's transition probabilities and that this asymmetry is precisely the kind of asymmetry of the formalism that Russell was looking for.

The second argument that one might extract from Russell's remark appeals to the timereversal invariance of a theory's fundamental equations:

16. The fundamental equations of all mature physical theories are time-reversal invariant.

17. There is no place for an asymmetric notion of cause in the context of a theory with time-reversal invariant laws.

18. Therefore, there is no place for an asymmetric notion of cause in mature physical theories.

Both premises of this argument, too, are open to challenge. Quantum mechanics contradicts (16). Since the Schrödinger equation is first-order in time, it is manifestly not time-reversal invariant (see Callender 2000). The Schrödinger equation is invariant under the joint operation of time-reversal and complex conjugation (in the coordinate representation), but this fact does not undermine the 
conclusion that, as Callender puts it, "time in a quantum world is handed." Curiously, then—and perhaps somewhat surprisingly - certain anti-causal arguments are even less compelling in the context of quantum mechanics than in the context of a Newtonian and putatively deterministic physics.

But even if were to grant (16), the move from the time-reversal invariance of the dynamical laws to the claim that there is no room for causes in a theory with such laws is far from compelling. First, (17) papers over the distinction between the claim that the relation between cause and effect is asymmetric and the claim that it is temporally asymmetric, yet there are cases where we may want to take a cause to operate simultaneous with its effect. Newton's Second Law, for example, is often interpreted causally in that the external force acting on a body is taken to be the cause of the body's simultaneous acceleration. And it is not immediately obvious how the time-symmetry of Newton's law could have any bearing on the question whether the force at a time is properly thought of as asymmetric cause of the simultaneously occurring acceleration.

Yet someone who is inclined to think of Newton's Laws causally presumably would also hold that causally affecting the acceleration of an object has an effect on the object's future evolution, but not on its past evolution, and it is this idea, presumably, that is supposed to be undermined by the observation that Newton's equations are time-reversal invariant. The claim, to repeat, is that timesymmetric dynamical laws are incompatible with time-asymmetric causal relationships. The intuitive idea that an external force on an object is a contributing cause to the object's later states but not its earlier states is undermined, according to this argument, by the fact that the dynamical equations also have a solution for which initial and final state are exchanged (or more, precisely where initial and final states are replaced by the time-reversed final and initial state, respectively).

I see no reason, however, why a defender of causation in Newtonian physics should be moved by this argument. One might try to argue that the notion of causation is incoherent. But this, it is important to recall, is not the claim we are currently considering. The current argument grants the prima facie legitimacy of causal notions but maintains that it is impossible for a world governed by time-symmetric laws to be time-asymmetrically causal. That is, the present argument grants that we can conceive of a world that evolves causally but insists that there could be no causally evolving world that is governed by time-symmetric dynamical laws. It is unclear to me what an argument for this latter claim might be. It does not follow from the fact that a formalism is time-asymmetric that that formalism has to be interpreted causally. (Hence I do not want to suggest that the fact that Schrödinger's equation is not time-reversal invariant implies that it has to be interpreted causally.) And similarly, it does not follow from the fact that a formalism is time-symmetric that it cannot be interpreted as representing states of a causally evolving system. Independently of whether theories are construed syntactically or semantically, it seems to me that there can be no general argument of the kind suggested by Russell or van Fraassen to show that causal assumptions cannot form an integral part of theories in physics. 
There is one final kind of argument I want to consider, which offers perhaps the most promising anti-causal strategy. This argument does not question the possibility or conceivability of a causal world with time-symmetric dynamical laws, but maintains that we could never have good reasons to postulate a time-asymmetric causal dependence of future states on past states. Such an epistemological argument might either appeal to the general construction of a Hume world or to the time-reversal invariance of the fundamental equations of physics. According to the latter considerations, there is no place for causal assumptions in fundamental physics, because we could never have scientifically legitimate reasons for interpreting time-reversal invariant laws causally:

19. The fundamental equations of all mature physical theories are time-reversal invariant.

20. There can be no scientifically justified reasons to include causally asymmetric notions in the interpretive framework of a theory with time-reversal invariant laws.

21. Therefore, there can be no scientifically justified reasons to include causally asymmetric notions of cause in mature physical theories.

I can think of two possible responses to this last argument. (20) implies that the only possible justification for allowing causes into a theory would have to be one that arises from the character of a science's dynamical laws. But there are at least two other avenues through which one might try to support causal assumptions. First, one can argue that our experimental interactions with physical systems provides us with scientifically legitimate reasons for adopting a causal interpretation of a theory with time-symmetric dynamical laws. In many scientific experiments we are concerned with systems that are closed except for localized experimental interventions. The goal of many experiments is to determine how different interventions into a system affect the system's evolutionthat is, how effects of the intervention percolate through the system. One general feature of all such interventions appears to be that they can affect the future evolution of a system but not its past. All of our interventions into experimental systems are 'from the past,' as it were. There are different ways in which this experimental asymmetry manifests itself: For example, we can prepare an initial state of a system and then calculate the system's future evolution from the initial state and the dynamical laws, but we cannot prepare a system's final state and use this state together with the laws to calculate the system's past evolution. Also, two experimenters can, without knowledge of the other's actions, prepare a system's initial state and engage in a later localized intervention into the system; but two experimenters cannot similarly set up the system's final state and an earlier intervention independently of each other.

This asymmetry is also related to a counterfactual asymmetry. Consider a system that is closed expect for a single intervention. Corresponding to different counterfactual interventions there are possible 'worlds' diverging from the actual world, in which the system begins in its actual initial state but ends up in final states different from its actual final state. But there also are possible 'worlds' in which the system begins in a non-actual initial state and after a non-actual intervention ends up in the actual final state. So far, then, there is no asymmetry. Yet there is a sense in which 
worlds with initial states identical to that of the actual world and non-actual final states are experimentally closer to the actual world than worlds with final states identical to the actual final state but non-actual initial states. We can experimentally probe counterfactual claims about a system by setting up replicas of a system that instantiate the counterfactual states in question. And it is a fact about our experimental interactions with such systems that it is in general much easier to set up multiple systems with identical initial states but different subsequent interventions than it is to set up systems that evolve into identical final states despite different prior interventions. As a concrete example consider a system consisting of a charged object in an external electromagnetic field. We are given the initial and final states of the field and the charge and are asking what the effects of interventions on the motion of the charge are. It is relatively easy to set up different systems in the same initial state characterized, say, by zero incoming fields and then intervene differently on the motion of the charge and accelerate it by different amounts. It is much harder to make sure that the final fields for several different systems are the same, even though the systems' charges are accelerated by different amounts (and hence are associated with different radiation fields.) Thus, our experimental interventions exhibit a temporal asymmetry and recent interventionist accounts of causation (for example, Woodward 2003) support the claim that this asymmetry may indeed be best thought of as a causal asymmetry.

A second, and perhaps related, observation that might lead us to posit time-asymmetric causal notions in a world with time-symmetric laws is that even a world with time-symmetric dynamical laws can exhibit time-asymmetric features and that there can be an asymmetry between the initial and final conditions characterizing typical systems in that world - as indeed is the case in the actual world. One example of this is the temporal asymmetry characteristic of wave phenomena in the presence of wave sources. This asymmetry intuitively consists in the fact that there are coherently diverging waves in nature but not their time-reverse - coherently converging waves - even though the dynamical laws governing waves are time-symmetric. One can show that this asymmetry ultimately consists in an asymmetry between prevailing initial and final conditions - roughly, the fact that incoming waves are often approximately equal to zero, while outgoing waves in the presence of wave sources are generally non-zero. One possible explanation for this asymmetry, which I defend in more detail in (Frisch 2005 and 2006a), is that wave sources asymmetrically cause disturbances propagating into the future.

Thus, ultimately I want to maintain that it is plausible that both the asymmetry of intervention and certain asymmetries characterizing prevailing initial and final conditions are best thought of as being grounded in a causal asymmetry. But there is not enough space here to defend this claim in detail and my aim in this paper is more modest. The fact that there are asymmetries that do not manifest themselves in asymmetries of the dynamical laws and the fact that these asymmetries can be given a causal explanation undermines an anti-causal argument purely from the time-reversal invariance of the dynamical laws. Thus, what I have argued here is that there are reasons distinct 
from considerations appealing to the form of the dynamical equations that can ground causal claims. This does not yet settle the question whether an appeal to causal considerations provides the best explanation of the asymmetries, but it does challenges the causal skeptic to provide an explanation for these asymmetries that are superior to a causal account. ${ }^{6}$

\section{Causal Reasoning in Physics: Dispersion Relations}

For all that I have said so far, one might still worry that a causal interpretation of a mature physical theory has the character of an alien element that is externally grafted onto the theory. The notion of cause, one might worry, is too crude and imprecise a tool in the context of the very precise functional dependencies with which a mathematical formalism presents us. Thus, (Norton 2007) argues that to the extent that causal notions play a useful role, it is as part of a "folk science" that does not represent the world with the same precision as our mature sciences do, while (Hitchcock 2007) argues that causal notions play a role in the more fundamental sciences only as provisional and imprecise guides to future research. In support of his view Hitchcock cites an earlier discussion by Patrick Suppes, who maintains that the notion of cause can play a legitimate role in science, but only as long as the phenomena at issue "have not yet succumbed to comprehensive mathematical analysis" that provide a "more precise characterization"- that is, only until it is "possible to apply simple fundamental laws to derive exact relationships such as those expressed in differential equations" (Suppes 1970).

One of the examples of the use of causal notions in physics as allegedly preliminary and imprecise tool that Suppes mentions in a passage quoted by Hitchcock is the role of causality constraints in the derivation of dispersion relations. Neither Suppes nor Hitchcock, however, offer any explanation as to how this example might support their view. In this section I want to look at this particular case in some more detail and argue that, far from supporting Hitchcock's and Suppes's conclusion, the derivation of dispersion relations is an example of how causal assumptions can play a substantive role in a mathematically precise treatment of physical phenomena.

Dispersion relations, which cover a wide range of scattering phenomena in many fields of physics, relate the absorptive properties of a medium to its dispersive (i.e. frequency-shifting) properties. If the absorptive properties of a medium are frequency dependent, then the selective absorption of parts of the wave will be accompanied by shifts in the shape of the wave. One particular instance of this is the dispersion of light in a dielectric medium characterized by a complex dielectric constant $\varepsilon$. The imaginary part of $\varepsilon$ characterizes the absorption of waves while the real part characterizes the phase shift and the dispersion relations specify how the two parts are related to each

\footnotetext{
${ }^{6}$ I take it that David Albert's and Barry Loewer's entropy accounts of causal counterfactuals are responses to this challenge (Albert 2000; Loewer 2007). I criticize their accounts in (Frisch 2006b; 2007; forthcoming) arguing that their attempts to ground the causal or counterfactual asymmetry in a Boltzmannian account of thermodynamics fail.
} 
other. Here is a brief sketch, following (Jackson 1975), of how dispersion relations are derived in this case. $^{7}$

The dielectric constant relates an incoming electric field $\mathbf{E}$ to the outgoing field, the electric displacement D:

$$
\mathbf{D}(\mathbf{x}, \omega)=\varepsilon(\omega) \mathbf{E}(\mathbf{x}, \omega)
$$

where $\varepsilon$ is the dielectric constant and $\omega$ the frequency of the wave. This frequency-dependent expression can, via Fourier's theorem, be transformed into a time-dependent expression for the displacement and field strengths:

$$
\mathbf{D}(\mathbf{x}, t)=\mathbf{E}(\mathbf{x}, t)+\int_{-\infty}^{+\infty} G(\tau) \mathbf{E}(\mathbf{x}, t-\tau) d \tau
$$

Here $G(\tau)$ is the Fourier transform of the dielectric constant and specifies the response to a unit pulse at time $\tau$. (2) is the most general mathematical relation that can be written down, for a system that is not explicitly time-dependent, for two fields for which there is a superposition principle - that is, for a primary field $\mathbf{E}$ and an output field $\mathbf{D}$ that is a linear functional of the input. One obvious feature of (2) is that it is non-local in time - that is, $\mathbf{D}(\mathbf{x}, t)$ depends on the value of $\mathbf{E}$ not only at $t$, but at all other times as well — and that, in particular, $\mathbf{D}$ depends on values of $\mathbf{E}$ at future times. The dispersion relations are derived by imposing an additional physically motivated constraint on the relation between incoming field and response field. The constraint is, as John Toll puts it in his seminal paper on dispersion relations, "Causality and the Dispersion Relations: Logical Foundations", that "no output can occur before the input." There should be no contribution from the integral on the right hand side of (2) for negative values of $\tau$ - that is, from electric field values in the future of $t$ - and (2) becomes:

$$
\mathbf{D}(\mathbf{x}, t)=\mathbf{E}(\mathbf{x}, t)+\int_{0}^{+\infty} G(\tau) \mathbf{E}(\mathbf{x}, t-\tau) d \tau
$$

The equality of the right-hand sides of (2) and (3) for arbitrary incoming electric fields implies

$$
G(\tau)=0 \quad \text { for } \tau<0 .
$$

The dispersion relations relating the real and imaginary parts of the dielectric constant $\varepsilon$ to each other follow as a mathematical theorem from the fact that the Fourier transform of $\varepsilon, G(\tau)$, vanishes for negative $\tau$. The precise form of these relations need not concern us here.

We can see why, intuitively, imposing the causality condition ought to imply a relation between the frequency- or shape-shifting properties of a medium and its absorptive properties. An incoming wave that is zero at all times before the time $t=0$ can be decomposed into Fourier components - a large number of sine and cosine waves - each of which extends from $t=-\infty$ to $t=+\infty$

\footnotetext{
${ }^{7}$ For a more general derivation of dispersion relations from a causality assumptions, see (Toll 1956).
} 
but which destructively interfere for all times $t<0$. Thus, if a medium were selectively to absorb a component of a certain frequency of an incoming wave without shifting the frequencies of the remaining components, then there would have to be an output wave containing the complement of the absorbed wave even before the arrival of the incoming wave. The causality condition denies that this is possible. Hence the condition implies that selective absorption has to be accompanied by a shifting of the frequencies of the remaining components. Without imposing this condition, of course, no constraint on the relation between the real and imaginary parts of the dielectric constant can be derived.

Thus, the overall structure of the derivation is this. We begin by positing a general mathematical relation between incoming and outgoing fields, assuming only linearity and no explicit time-dependence. We then appeal to an additional physical constraint on the relation between the fields - the causality condition - which further constrains the relation between the two fields. And it is this constraint that then allows us to derive the dispersion relation. The additional constraint, Jackson tells us, is "in accord with our fundamental ideas of causality in physical phenomena. Jackson shows that one can in fact derive (3) from (2) for a particular simple model for the index of refraction of a medium, but insists that (3) is "the most general spatially local, linear, and causal relation that can be written between $\mathbf{D}$ and $\mathbf{E}$ in a uniform isotropic medium" and that "its validity transcends any specific model of $\varepsilon(\omega)$," (309) such as the one discussed by him. Thus, the dispersion relations "are of very general validity, following from little more than the assumption of the causal connection [(3)] between the polarization and the electric field." (311) The relations, Jackson adds, are "extremely useful in all areas of physics. Their widespread application stems from the very small numbers of physically well-founded assumptions necessary for their derivation." (312))

Norton argues that a defender of a role for causal notions in science faces the following dilemma. Either causal assumptions place a factual constraint on the content of a science. But, Norton maintains, any candidate for such a factual constraint turns out to be false. Or causal assumptions place no restrictions on the content of a science. But then causal notions are nothing but an empty honorific. How does this dilemma apply to the present case? It is common (and perhaps near universal) practice to call the condition that there is no contribution to the outgoing wave from an incoming wave in its future a "causality condition". And it seems that this label is more than an empty honorific. (2) specifies a general mathematical relation between incoming and outgoing fields and there seems to be no formal or mathematical reason for any further constraint on the integral or the function $G(\tau)$. If however we think of the incoming wave as causing, as 'being responsible for', or as 'generating' the outgoing wave, then the causal asymmetry provides a physical motivation for the additional constraint. Thus, interpreting the relation between the $\mathbf{E}$ - and $\mathbf{D}$-fields causally has factual consequences and the causal constraint is, as Jackson puts it, "physically well-founded."

Does our taking the assumption to be well-founded commit us to its truth? Norton's strategy in arguing for the claim that there is no non-empty 'principle of causality' is to present 
counterexamples from different domains of physics to each putative candidate for such a principle. Thus, Norton must assume that a causal principle can play a legitimate role in any scientific theory only if we have good reasons to believe in its truth or its universal applicability. I think that this assumption is much too strong. It might in fact be true that effects never precede their causes. But I think that we can allow for the possibility that a certain causal principle or assumption is not true in general and nevertheless take it to be physically well-founded, in a certain sense. Causal assumptions - just as other scientific assumptions — can play a substantive and legitimate role in a certain domain — and their use can be physically well-founded in that domain—even if we take the assumptions to have only a limited domain of application and we in fact believe that the assumptions fail outside of that domain. It would not undermine Jackson's claim that the assumption of forward causal dependence is physically well-founded in the context of scattering phenomena, if we were to discover backward causation in another domain.

A defender of causal notions in science need not be committed to the view that if causal conditions place factual constraints on scientific theorizing, they must function as prior metaphysical constraints on all theorizing. Rather, such conditions can function as scientific constraints of varying universality or generality. The time-asymmetric assumption that effects do not precede their causes may well have universal scope - tellingly, Norton's list of counterexamples does not include a counterexample to this principle - but other putatively causal constraints may have a more restricted scope. Thus, causal locality conditions may be physically well-founded and play a legitimate role in some theories, such as classical electrodynamics, even if we believe that this assumption is violated in Newtonian gravitational theory. We could not be committed to the truth of a universal locality principle, if we also believed - as we in fact do not - that Newton's law of gravitation is true. Yet we can accept that a causal locality principle plays a legitimate role in some mature sciences, even if we believe that no such principle is true in general. Thus, if it were indeed the case that there is no "principle of causality" that does not fail in some domain or other, as Norton argues, then this might show that causal fundamentalism is false, but it does not follow that no causal principle can play a substantive role in a mature science. ${ }^{8}$

I want to end this section with a brief remark concerning the derivation of dispersion relations in quantum field theory, governing the scattering of photons or other spin-zero particles (see, for example, Gell-Mann 1954). Here, too, the dispersion relations are derived from a principle that is commonly called a "causality condition." The only difference to the classical case that is relevant to our discussion is that this condition has two distinct components. The first component is the

\footnotetext{
${ }^{8}$ Norton contrasts putative principles of causality with the principle of conservation of energy, which he claims is a principle "to which all physical theories must conform" (Norton forthcoming, 231). Yet arguably the roles of the two kinds of principles are more closely analogous than Norton suggests. Models of general relativity do not all permit the formulation of a principle of global energy-momentum conservation. Yet this does not undermine the fact that the principle is physically well-founded and can play an important role in the context of other theories.
} 
relativistic condition of 'micro-causality,' which (for boson fields) is the requirement that the commutator for two field operators taken at spacelike separated points should vanish. This requirement alone imposes a locality constraint and is time-symmetric. The second component is a time-asymmetric constraint according to which the scattered field only depends on the incident field in is past. Together, these two requirements restrict the dependence of the scattered field on the incident field to the past lightcone. Again, these two conditions can be expressed mathematically precisely:

$$
<0\left|\left[\phi(x), \phi\left(x^{\prime}\right)\right]\right| 0>\eta\left(x-x^{\prime}\right)=0 \text {, if }\left(x-x^{\prime}\right)^{2}=0 \text { or if } x_{0}<x^{\prime}{ }_{0} .
$$

The expression in brackets is the expectation value of the field commutator and $\eta(x)$ is a step function that is equal to zero for $x_{0}<0$ and is equal to one for $x_{0} \geq 0 .{ }^{9}$ Thus, we here see two of the aspects of causal notion that I distinguished in section 2 invoked jointly: the causal condition imposes both a locality constraint and a time-asymmetric constraint. A time-asymmetric causal assumption, according to which the interaction between incoming fields and scatterer results in a scattered field, also appears to be implicit in the way scattering is modeled, where one assumes that the outgoing spherical wave is the result of an incident plane wave interacting with the scattering center.

\section{Conclusion}

I distinguished three uses of causal notions in physics, expressing a principle of determinism, imposing locality conditions, and expressing time-asymmetric constraints. I focused on the third usage and examined a range of arguments that have been advanced against the claim that an asymmetric notion of cause can play a role in mature physical theorizing. None of these anti-causal arguments, I argued, are cogent. Finally, I argued that the role played by time-asymmetric causal assumptions in the derivation of dispersion relations is evidence for the fact that asymmetric causal notions richer than what Russell would have allowed continue to play an important role in physics.

${ }^{9} x_{0}$ is the time-coordinate of the spacetime point $x$. 


\section{References}

Albert, David Z. 2000. Time and Chance. Cambridge, Mass.: Harvard University Press.

Bohr, Niels. 1948. On the Notions of Causality and Complementarity. Dialectica 2: 312-319.

Callender, Craig. 2000. Is Time 'Handed' in a Quantum World? Proceedings of the Aristotelian Society, June, 247-269.

Cartwright, Nancy. 1993. Defence of 'This Worldly' Causality: Comments on van Fraassen's Laws and Symmetry. Philosophy and Phenomenological Research 53 (2): 423-429.

Frisch, Mathias. 2005. Inconsistency, Asymmetry and Non-locality: A Philosophical Investigation of Classical Electrodynamics. New York: Oxford University Press.

_.2006a. Causal Asymmetry, Counterfactual Decisions and Entropy. Philosophy of Science 72 (5):739-750.

-2006b. A tale of two arrows. Studies in the History and Philosophy of Modern Physics 37

(3):542-558.

2007. Causation, Counterfactuals and the Past-Hypothesis. In Russell's Republic: The Place of Causation in the Constitution of Reality, edited by H. Price and R. Corry. Oxford: Oxford University Press.

Gell-Mann, M., M. L. Goldberger, W. E. Thirring. Use of Causality Conditions in Quantum Theory. Physical Review 95 (6): 1612-1627.

Hitchcock, Cristopher. 2007. What Russell Got Right. In Causality, Physics, and the Constitution of Reality: Russell's Republic Revisited, edited by H. Price and R. Corry. Oxford: Oxford University Press.

Jackson, John David. 1975. Classical Electrodynamics. 2nd ed. New York: John Wiley \& Sons. Lange, Marc. 2002. An Introduction to the Philosophy of Physics: Locality, Fields, Energy, and Mass. Oxford: Blackwell Publishers.

Loewer, Barry. 2007. Counterfactuals and the Second Law. In Causality, Physics, and the Constitution of Reality: Russell's Republic Revisited, edited by H. Price and R. Corry. Oxford: Oxford University Press.

Norton, John D. 2007. Causation as Folk Science. In Causality, Physics, and the Constitution of Reality: Russell's Republic Revisited, edited by H. Price and R. Corry. Oxford: Oxford University Press.

Norton, John D. forthcoming. Do the Causal Principles of Modern Physics Contradict Causal AntiFundamentalism? In Causality: Historical and Contemporary, edited by P. K. Machamer and G. Wolters. Pittsburgh: University of Pittsburgh Press.

Rohrlich, Fritz. 1990. Classical Charged Particles. Reading, MA: Perseus Books.

Russell, Bertrand. 1918. On the Notion of Cause. In Mysticism and Logic and other Essays. New York: Longmans, Green and Co.

Schrödinger, Erwin. 1951. Science and Humanism. Cambridge: Cambridge University Press. 
Steiner, Mark. 1986. Events and Causality. The Journal of Philosophy 83 (5): 259-264.

Suppes, Patrick. 1970. A Probabilistic Theory of Causality. Amsterdam: North-Holland.

Toll, John S. 1956. Causality and the Dispersion Relation: Logical Foundation. Physical Review 104 (6):1760-1770.

Van Fraassen, Bas. 1993. Armstrong, Cartwright, and Earman on Laws and Symmetry. Philosophy and Phenomenological Research 53 (2): 431-444.

Weyl, Hermann. 1989. The Open World. Woodbridge: Ox Bow Press. Original edition, 1932.

Woodward, James. 2003. Making Things Happen: A Theory of Causal Explanation. Oxford: Oxford University Press.

- 2007. Causation with a Human Face. In Causality, Physics, and the Constitution of Reality: Russell's Republic Revisited, edited by H. Price and R. Corry. Oxford: Oxford University Press. 